\title{
Inhibition of thrombin-induced platelet aggregation using human single-chain Fv antibodies specific for TREM-like transcript-I
}

\author{
Barbara Giomarelli',, Valance A. Washington 2,3,*, Maia M. Chisholm², Laura Quigley², James B. McMahon', \\ Toshiyuki Moril'\#, Daniel W. McVicar ${ }^{2}$ \\ 'Molecular Targets Development Program, ${ }^{2}$ Cancer and Inflammation Program- Center for Cancer Research, National Cancer \\ Institute, NCl-Frederick, Frederick, Maryland, USA; ${ }^{3}$ Department of Anatomy and Cell Biology, Universidad Central del Caribe, Bayamón, \\ Puerto Rico
}

\section{Summary}

TREM-like transcript-I (TLT-I) is a novel platelet membrane receptor, which has been recently characterized in mice. TLT-I is expressed exclusively in platelets and megakaryocytes, and its expression is dramatically upregulated upon platelet activation, suggesting that it plays a unique role in hemostasis and/or thrombosis. In this study we identified and characterized highly specific human monoclonal antibodies that bind to TLT-I by screening a naïve library of single chain Fv fragments (scFvs) displayed on filamentous phage (Thomlinson I library). These scFvs

\section{Keywords}

Triggering receptors expressed on myeloid cells (TREM)-like transcript-I (TLT-I), anti-thrombosis therapy, human antibodies, single chain fragments (scFvs), phage display

\section{Introduction}

Platelets play a crucial role in the mortality associated with cardiovascular disease (CVD) and therefore are a primary target for therapeutic intervention. Platelets are believed to assist in the advancement of CVD (1), and it is estimated that $50-60 \%$ of sudden coronary death cases are caused by ruptured plaques consisting largely of platelets and fibrin $(2,3)$. To improve patient outcomes, drugs targeting platelet agonists are commonly used. Current efforts in anti-platelet therapy focus on separating the haemostatic function of platelets from their thrombotic nature $(4,5)$. Some drugs have been designed to specifically inhibit receptors on platelets. Examples are clopidogrel, which inhibits platelet activation through the irreversible inhibition of the $\mathrm{P}_{2} \mathrm{Y}_{12}$ receptor (6) and abciximab, a monoclonal $\left.\mathrm{F}(\mathrm{ab})_{2}\right)_{2}$ fragment that targets the platelet glycoprotein (GP) IIb/IIIa $(7,8)$. Al- detected plate-bound TLT-I, captured soluble TLT-I, and readily reacted with cell-bound TLT-I on transfectants and primary human platelets. Most importantly, anti-TLT-I scFvs inhibited thrombin-mediated human platelet aggregation. This inhibition was specific for thrombin-induced aggregation and was reversible with higher doses of agonist. These data are the first to demonstrate a biological role for TLT-I and its potential as a therapeutic target. The human scFvs isolated in this study may represent novel anti-platelet therapeutic agents.

Thromb Haemost 2007; 97: 955-963

though these therapies have shown partial efficacy and side effects, they demonstrate the utility of platelet membrane receptors as a new class of therapeutic targets for the regulation of platelet functions.

We recently cloned and characterized a receptor called TREM-like transcript-1 (TLT-1) from both mice and humans. We demonstrated that: i) murine TLT-1 (mTLT-1) is abundantly expressed only on platelets and megakaryocytes, and ii) both mTLT-1 and human TLT-1 (hTLT-1) are sequestered in the platelet alpha granules and quickly translocated to the platelet surface upon platelet activation $(9,10)$. The specificity and regulation of TLT-1 expression suggests that it plays a unique role in vascular homeostasis, making it an attractive tool for the dissection and manipulation of platelet function (10). TLT-1 is a member of the triggering receptors expressed in myeloid cells (TREMs), a family of proteins that play important roles in the 
regulation of both adaptive and innate immunity (11). Murine TLT-1 contains a single V-set Ig type-extracellular domain and a transmembrane region that lacks charged amino acids unlike other members of the TREM cluster. The cytoplasmic domain contains two cytoplasmic tyrosine residues, one of which conforms to the consensus described for an immunoreceptor tyrosine-based inhibitory motif (ITIM) $(9,12)$. Consistent with the fact that both human and mouse proteins contain cytoplasmic ITIMs expected to bind src-homology phosphotases (SHP), two groups have demonstrated such binding $(9,12)$. Interestingly, whereas we have demonstrated the ability of TLT-1 to bind SHP-1 when phosphorylated (9), TLT-1 interactions with SHP-2 have also been detected, and co-ligation suggested that hTLT-1 enhances $\mathrm{Ca}^{2+}$ mobilization in response to Fc receptor-crosslinking, denoting potential co-activator rather than inhibitory function (12). Co-activation function has not been tested in mice, and the relevance of these findings to any function in platelets remains to be demonstrated.

Our understanding of TLT-1 function has been in part limited by the lack of suitable tools to dissect TLT-1's role in platelet-mediated thrombosis and homeostasis. In this study we sought to assess TLT-1's potential as a therapeutic target in the treatment of thrombotic diseases using monoclonal antibodies directed against functional epitopes. Phage display of single chain Fv $(\mathrm{scFv})$ offers a new way to produce monoclonal antibodies with defined binding specificities $(13,14)$. ScFv antibodies contain the variable regions of heavy and light chains connected by a linker peptide and represent the smallest units retaining the antigen-binding specificity of whole IgGs (15). Importantly, when these antibody fragments are of human origin, adverse immune responses in human therapy can be minimized $(16,17)$.

In this study, we used a naïve phage-displayed library to isolate a panel of human scFvs that are highly specific for TLT-1 and used them to identify a functional role for TLT-1 in platelet aggregation. Specifically, we found that targeting TLT-1 with the scFvs resulted in reduced thrombin-induced aggregation of human platelets. We found that the scFvs differentially affected platelet aggregation and that this inhibition was dose-dependent. The potential of these scFvs for the treatment of thrombosis and other platelet-related diseases is discussed.

\section{Materials and methods}

\section{Antibody library and bacterial strains}

The Human Single Fold scFv library Tomlinson I used in this study was provided from the Medical Research Council (MCR Geneservices, Cambridge, UK; reference at http://www.gene service.co.uk/products/proteomic/scFv_tomlinsonIJ.jsp). The library was amplified as previously described (18) and consisted of a repertoire of $1.47 \times 10^{8}$ different antibody clones. Helper phage KM13 and Escherichia coli HB2151 were also provided by MRC. E. coli TG1 was purchased from Stratagene (La Jolla, CA, USA).

\section{Panning of the library}

Panning of the library was performed in immunotubes (Maxisorp, Nunc, Gaithersburg, MD, USA) as previously described (18). Briefly, tubes were coated with a purified fusion of the extracellular domain of TLT-1 with the Fc fragment of human IgG1 (TLT-1-Fc) (10) $(10 \mu \mathrm{g} / \mathrm{ml}$ for the first and second round, and $1 \mu \mathrm{g} / \mathrm{ml}$ for the third round) or with $\mathrm{Fc}$ fragment (Bethyl, Montgomery, TX, USA; $100 \mu \mathrm{g} / \mathrm{ml}$ ) in phosphate buffer saline (PBS), washed with PBS, and blocked with $2 \%$ skimmed milk in PBS (MPBS). All incubations of phage in immunotubes were at room temperature (RT) for 2 hours (h) in a total volume of $1 \mathrm{ml}$. A subtraction step was used to deplete the members of library that bind to the Fc fragment part of TLT-1-Fc. Phage (approximately $2 \times 10^{12}$ transducing units, TU) were incubated with $2 \%$ MPBS in immunotubes coated with Fc fragment. The subtracted library was then incubated in immunotubes coated with TLT$1-\mathrm{Fc}$. Fc fragment $(10 \mu \mathrm{g} / \mathrm{ml})$ was also added to the solution during the selection to further remove the library members that reacted with $\mathrm{Fc}$. Unbound phage were removed by washing with PBS + 0.1\% Tween 20 (PBS-T). Bound phage were eluted by trypsin (Sigma, St. Louis, MO, USA) and rescued by reinfection of E. coli TG1 as previously described (18). Rescued phage were used for the next round of selection. Three rounds of selection were carried out.

\section{Selection of anti-TLT-I phage clones}

Individual clones were randomly picked out from the phage pools of three rounds of selection. Phage were produced and used for monoclonal phage ELISA as previously described (19). Briefly, 96-well plates (Maxisorp Nunc-Immunoplates, Nunc) were coated with TLT-1-Fc (100 ng/well in PBS) or with Fc fragment $(1 \mu \mathrm{g} / \mathrm{well})$ and blocked with MPBS. A control plate was incubated with MPBS only. The supernatants containing scFvphage were incubated in the TLT-1-Fc-coated plate and in the control plates. Phage binding was detected with HRP-conjugated anti-M13 antibody (Amersham Bioscience, Piscataway, NJ, USA). Three washes with PBS-T (200 $\mu$ l) were performed at each step. The specificity of binding to TLT- 1 for the selected clones was further confirmed by phage ELISA with bovine ubiquitin (Sigma, St. Louis, MO, USA), and mouse activating receptor 1 (MAR-1), a gift from Dr. John Ortaldo (NCI-Frederick). An irrelevant scFv phage clone (negative clone 1) that did not bind to TLT- 1 and control antigens was isolated from the same library and used as a negative control in our experiments.

\section{Sequencing of the selected clones}

The scFv insert of individual clones was amplified using a highfidelity polymerase Pfu (Platinum PCR Supermix, Invitrogen) with the following primers: LMB3 (5'- CAGGAAACAGCTATGAC $-3^{\prime}$ ) and pHENseq (5'- CTATGCGGCCCCATTCA -3'). Primers used for sequencing of the PCR products were: Link-seqnew (5'-CGACCCGCCACCGCCGCTG -3') and pHENseq.

\section{Expression and purification of soluble scFvs}

Production of soluble scFvs was performed in the non-suppressor E. coli strain HB2151 as previously described $(19,20)$. Expression of scFvs was induced by adding $1 \mathrm{mM}$ isopropyl$\beta$-D-thiogalactopyranoside (IPTG) when the optical density at $600 \mathrm{~nm}\left(\mathrm{OD}_{600}\right)$ of the $\mathrm{HB} 2151$ cultures was 0.9 and growing with shaking $(250 \mathrm{rpm})$ for $16 \mathrm{~h}$ at $30^{\circ} \mathrm{C}$. Supernatants were subjected to SDS-PAGE in a Tris-glycine $8-16 \%$ gel and proteins were transferred to a polyvinylidene difluoride (PVDF) mem- 
brane according to standard procedures. The membrane was blocked with 3\% MPBS and probed with HRP-conjugated antipentahistidine (His-tag) antibody (Qiagen, Hilden, Germany). For purification of scFvs, supernatants were filtered through a $0.2 \mu \mathrm{m}$-pore size membrane (Corning, NY, USA) and subjected to ammonium sulfate precipitation at $70 \%$ saturation $\left(4^{\circ} \mathrm{C}\right)$. The resulting precipitates were dissolved and dialyzed in PBS. scFvs were purified by Protein L columns (Pierce, Rockford, IL, USA) according to manufacturer's instructions. Purified scFvs were dialyzed in PBS at $4^{\circ} \mathrm{C}$ for $16 \mathrm{~h}$. Purity of the scFvs was evaluated by SDS-PAGE. An irrelevant $\mathrm{scFv}$ was expressed from negative phage clone 1 and purified as described above. This $\mathrm{scFv}$ was used as a negative control in the experiments of $\mathrm{scFv}$ binding to TLT- 1 and in the platelet aggregation experiments.

\section{scFv ELISA}

The binding activity of soluble scFvs to TLT- 1 was analyzed in an ELISA assay with two different modalities. For standard ELISA, TLT-1-Fc or Fc fragment were immobilized at $1 \mu \mathrm{g} /$ well onto 96-well plates. After blocking with PBS 3\% BSA for $3 \mathrm{~h}$ at RT, scFv-containing supernatants $(50 \mu \mathrm{l})$ or purified scFvs $(1 \mu \mathrm{g} / \mathrm{well})$ in $3 \% \mathrm{BSA}$ were incubated for $1 \mathrm{~h}$ at RT. Washes were incorporated at each appropriate step. Binding of scFvs was detected by Protein L-HRP conjugate (Sigma) diluted 1:5,000 in $3 \%$ BSA. The ability of the scFvs to bind TLT- 1 in solution was evaluated in a sandwich ELISA. A 96-well plate was coated overnight at $4{ }^{\circ} \mathrm{C}$ with $1 \mu \mathrm{g} /$ well of purified scFvs. After blocking with $3 \%$ BSA, serial dilutions of TLT-1-Fc (1:3) were added from $90 \mathrm{ng}$ to $1 \mathrm{ng} /$ well. Binding of TLT-1-Fc to the immobilized scFvs was evaluated by incubating with a rabbit anti-TLT-1 polyclonal antibody (pAb) $(1: 1,000)$ (10) followed by incubation with HRP-conjugated anti-rabbit IgGs (Sigma). Binding was measured as previously described (20).

\section{Cell culture and transfection}

Human embryonic kidney (HEK)293T cells were maintained and transfected as previously described (21). Human TLT-1 cDNA was generated by RT-PCR using Platinum HiFi-Supermix (Gibco-BRL, Grand Island, NY, USA) with human platelet cDNA as a template and cloned into pEF6V5-His TOPO (Invitrogen) using primers: forward (5'-ATGGGCCTCACCCTGCTCTTG-3') and reverse (5'-GCTGGATGGAGTCTGATTG-3'). Human venous endothelial cells were kindly provided by Giovanni Melillo (NCI Frederick). Cell purifications were completed as previously described (10).

\section{Preparation of platelets}

Fresh platelets concentrated in acid-citrate-dextrose were obtained from the NIH blood bank. Washed platelets were prepared as previously described (22). Briefly, platelets were isolated by centrifugation at 2,100 $\times \mathrm{g}$ for 10 minutes (min) and resuspended in Tyrode's solution $(2 \mathrm{mM} \mathrm{MgCl}, 137 \mathrm{mM} \mathrm{NaCl}, 2.68 \mathrm{mM}$ $\mathrm{KCl}, 3 \mathrm{mM} \mathrm{NaH}_{2} \mathrm{PO}_{4}, 0.1 \%$ glucose, $5 \mathrm{mM}$ HEPES, $\mathrm{pH} 7.35$ ) at a final concentration of $3.2 \times 10^{8} \mathrm{cells} / \mathrm{ml} . \mathrm{Ca}^{2+}$ and aparase were added at final concentration of $2 \mathrm{mM}$ and $0.02 \mathrm{U} / \mathrm{ml}$, respectively. To prepare platelet-rich plasma (PRP), whole blood was collected in acid citrate dextrose (1:7) from healthy volunteers and centrifuged at $800 \mathrm{xg}$ to for $20 \mathrm{~min}$ to remove red blood

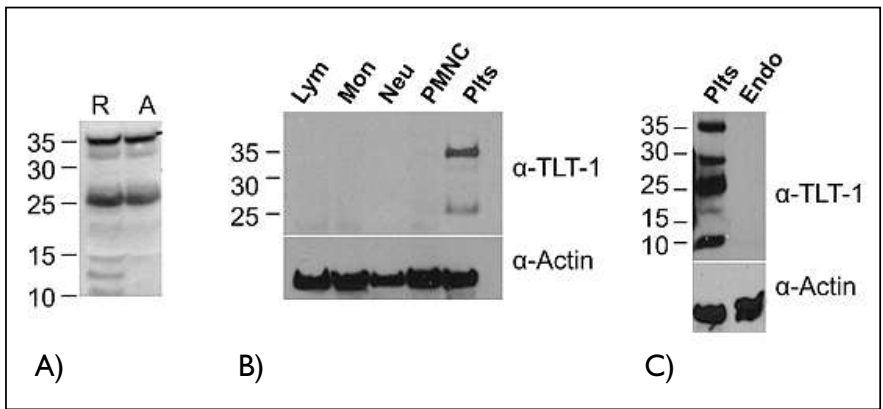

Figure I:TLT-I expression in the peripheral blood. A) Expression of TLT- $I$ in human platelets. Western blot analysis of TLT-I expression using $10 \mu \mathrm{g}$ of resting $(\mathrm{R})$ or activated $(\mathrm{A})$ platelets. A commercial antiTLT-I polyclonal antibody (pAb) was used for TLT-I detection. B) TLT-I is only expressed in platelets. Peripheral blood populations were separated, and whole cell lysate $(\mathrm{WCL})$ of $2 \times 10^{6}$ cells were analyzed by Western blot for TLT-I expression probing with anti-TLT-I pAb. C) TLT-I is not expressed in endothelial cell lines. Western blot using human venous endothelial cells (Endo) and platelets (plts) WCL probed with anti-TLT-I pAb.

cells. Half of the collected PRP was spun at 2,100 $\mathrm{x} g$ to remove platelets, and the resulted platelet-poor plasma was used to dilute the PRP to $3.2 \times 10^{8}$ platelets $/ \mathrm{ml}$ with the addition of aparase (0.02 units, $\mathrm{U} / \mathrm{ml})$.

\section{Flow cytometry}

For flow cytometric analysis with TLT-1-transfected cells, phage-containing supernatants were prepared from individual colonies as previously described (14). (HEK)293T-TLT-1 transfected cells $\left(2 \times 10^{5}\right.$ cells) were washed with PBS $1 \%$ BSA and incubated with phage $\left(2 \times 10^{10} \mathrm{TU}\right)$ or purified $\mathrm{scFv}(1 \mu \mathrm{g} / \mathrm{ml})$. After $1 \mathrm{~h}$ of incubation at $4^{\circ} \mathrm{C}$, cells were washed twice with $\mathrm{PBS} / 1 \%$ BSA. Phage binding was detected by incubation with mouse anti-M13 monoclonal antibody (Amersham Bioscience, Piscataway, NJ, USA), followed by incubation with phycoerithrin (PE)conjugated anti-mouse antibody (Jackson Immunoresearch, West Grove, PA, USA). To analyze scFv binding to human platelets, platelets were incubated with $1 \mu \mathrm{g}$ of purified $\mathrm{scFv}$ for $1 \mathrm{~h}$ at $4^{\circ} \mathrm{C}$ and washed three times with PBS containing $0.1 \%$ sodium azide and $0.1 \%$ BSA (FACS buffer). ScFv binding was detected

Table I: Deduced amino acid sequences of CDR2 and CDR3 of $\mathbf{V}_{\mathbf{H}}$ and $\mathbf{V}_{\mathbf{L}}$ domains of the selected scFvs. Identical residues are denoted by (-). CDRs were identified based on Kabat definition (http://www.bioinf.org.uk/abs/).

\begin{tabular}{|l|l|l|l|l|}
\hline Clone & VH-CDR2 & VH-CDR3 & VL-CDR2 & VL-CDR3 \\
\hline Al & GIGTTGYATAYADSVKG & GNSGFDY & TASTLQS & QQSSTDPGT \\
\hline A7 & A-GSY-SA-A------ & NGYD--- & N--G--- & --SAAN-S- \\
\hline A8 & G-GTT-YS-A------ & TAYT--- & S------ & --NSTY-A- \\
\hline B8 & N-TAS-YA-A------ & TTAT--- & D--N--- & --DTAS-S- \\
\hline CI0 & G-SST-GA-T------ & STYD--- & G------ & --NADA-T- \\
\hline DI & A-GTT-YA-A----- & ANSY--- & S--T--- & --DSTS-D- \\
\hline
\end{tabular}


using a FITC-conjugated anti-c-myc antibody (Sigma). TLT-1 expression was detected with a commercial anti-human TLT-1 pAb (R\&D Systems, Minneapolis, MN, USA) as previously described (10). Resting platelets and scFv negative clone 1 were used as negative controls.
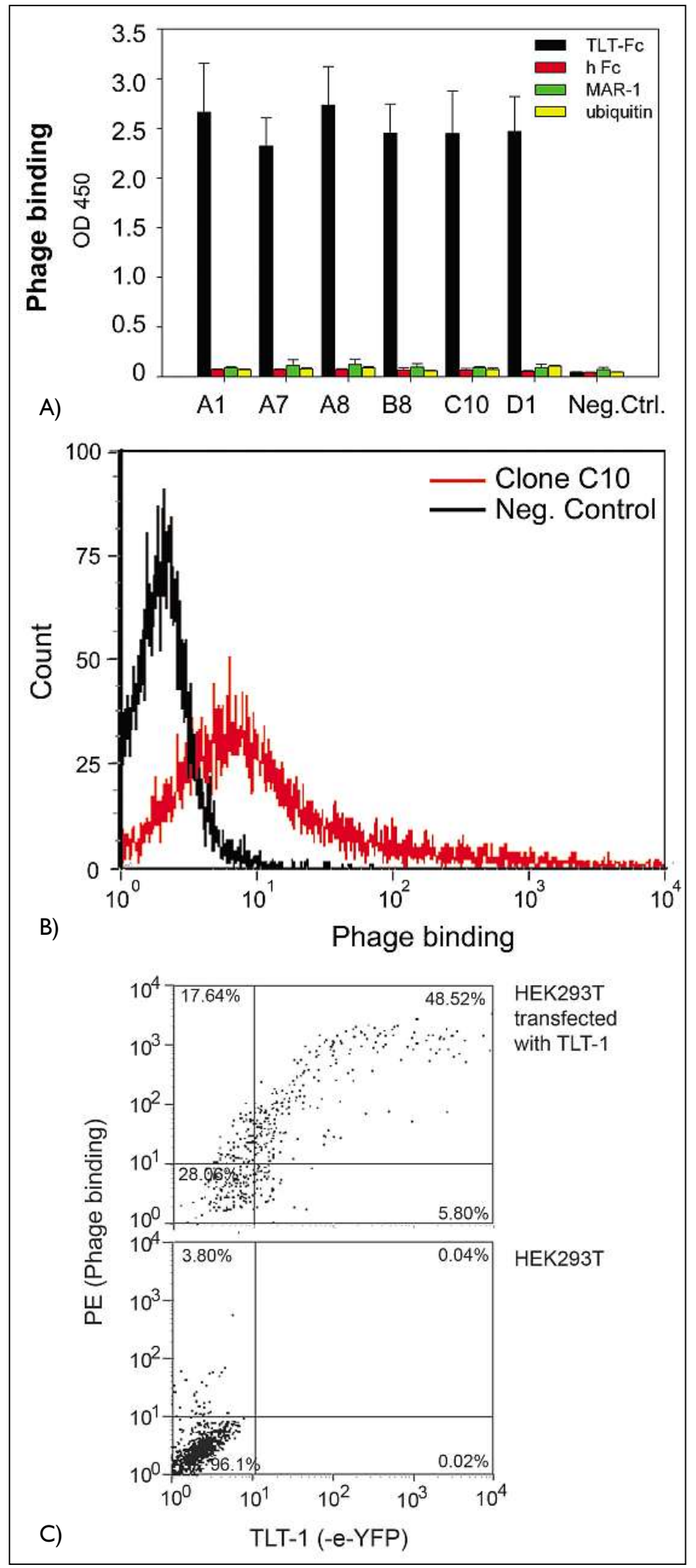

\section{Platelet aggregation}

Aggregation assays were run on a chronology aggregrometer (Chronolog, Havertown, PA, USA). ScFvs or vehicle (PBS) were added and allowed to incubate with the PRP or with washed platelets for 3 min at $37^{\circ} \mathrm{C}$ with stirring $(800 \mathrm{rpm})$ before adding the agonists. Thrombin $(0.125 \mathrm{U} / \mathrm{ml}$; Calbiochem, San Diego, CA, USA), collagen ( $5 \mu \mathrm{g} / \mathrm{ml}$, Chronolog), U46619 ( $7 \mu \mathrm{M}$; Cayman Chemical, Ann Arbor, MI, USA), phorbol myristate acetate (PMA, $10 \mu \mathrm{M}$; Calbiochem), adenosine diphosphate (ADP, $10 \mu \mathrm{M}$; Sigma) or convulxin $(20 \mathrm{nM})$ was added to the aggregation cuvette containing $600 \mu \mathrm{l}$ of platelet suspension $\left(3 \times 10^{8} / \mathrm{ml}\right)$, and results were recorded by a chart recorder for at least 5 min. Convulxin was kindly provided by Ivo Francischetti (NIH, Bethesda, MD, USA).

\section{Immunoprecipitation}

For immunoprecipitation (IP), platelets $\left(3 \times 10^{8} / \mathrm{ml}\right)$ in tyrodes buffer, containing $13 \mathrm{mM}$ ethylene glycol-bis( $\beta$-aminoethyl ether)N,N,N',N'-tetraacetic acid (EGTA) were incubated with or without thrombin $(1 \mathrm{U} / \mathrm{ml})$. Cells were lysed as previously described (10), and $2 \mu \mathrm{g}$ of purified scFv $\mathrm{C} 10$ or an irrelevant $\mathrm{scFv}$ (negative clone 1) was incubated with the whole cell lysate in a volume of $1 \mathrm{ml}$ with rotation at $4^{\circ} \mathrm{C}$ overnight. Fifty $\mu \mathrm{l}$ of Protein $\mathrm{L}$ beads, which were washed three times with PBS, were added per $\mathrm{ml}$ of lysate, and washed three times with $0.1 \%$ triton X-100 after rotating for $1 \mathrm{~h}$ at $4^{\circ} \mathrm{C}$. Proteins were eluted from the beads by boiling in SDS sample buffer. For immunoblotting eluted proteins were resolved by SDSPAGE on 7\% acrylamide gels. Immunoblotting was performed by transferring the proteins to a PVDF membrane and probing with anti-human TLT-1 pAb (R\&D Systems). Bound antibodies were detected using HRP-conjugated goat anti-rabbit IgG (Amersham Biosciences, Buckinghamshire, UK).

\section{Results}

\section{Expression and characterization of hTLT-I}

We have previously shown that murine TLT-1 (mTLT-1), which has an expected molecular weight of $34 \mathrm{kDa}$, is expressed as a $45 \mathrm{kDa}$-protein in peripheral blood platelets, likely due to glycosolation (10). To analyze the expression of various human TLT-1 (hTLT-1) isoforms in the peripheral blood and in different cell types, we used a goat anti-hTLT-1 polyclonal antibody (pAb) to probe whole cell lysate of resting or thrombin-activated platelets

Figure 2: Selection of phage antibodies that binds to TLT-I. A) Clones that specifically bound to TLT-I but not to the Fc fragment were isolated in a monoclonal phage ELISA format. For phage ELISA, TLT-I-Fc (black histograms), Fc fragment (red histograms), MAR-I (green histograms) or ubiquitin (yellow histograms) were immobilized on a microtiter plate $(10 \mu \mathrm{g} / \mathrm{ml})$ and incubated with purified phage preparations ( $10^{\prime \prime}$ phage). Binding was detected using an anti-MI 3 antibodyHRP conjugate. The negative control (Neg. Ctrl.) is the binding of the secondary antibody only. The data represented are the mean of three independent experiments. B, C) The isolated phage clones were analyzed by flow cytometry for binding to TLT-I transfected cells and to transfection control cells (HT293K). Phage from the naïve libraries were also used as a negative control ( Neg. $\mathrm{Cl}$.). The histograms (B) and dot blots (C) for clone $\mathrm{ClO}$ are shown. 
in Western blot analysis (Fig. 1A). We detected two major bands of 35 and $25 \mathrm{kDa}$, in both resting and activated platelets. The $35 \mathrm{KDa}$ species is full-length TLT- 1 and the $25 \mathrm{kDa}$ band corresponds to the TLT-1sp reported by Barrow et al. (12). A minor band of $33 \mathrm{kDa}$ was also detected in both resting and activated platelets, while three bands of 15,13 and $11 \mathrm{kDa}$ were visible in resting but not in activated platelets. These bands represent degradation products of TLT-1 (22, data not shown). The three lowest-molecular-weight species can also be found in serum or in cell culture supernatant of TLT-1 transfected cells (22). In mouse TLT-1 is only found in megakaryocytes and platelets (10). Our previous Northern analysis data on human peripheral blood subsets suggested that hTLT-1 might also be platelet specific (10). To evaluate this at protein level, we isolated lymphocytes, monocytes, neutrophils, polymorphonucleate cells (PBMCs), human vascular endothelial cells (HUVECs), and platelets and probed them with the anti-TLT-1 pAb in Western blot analysis (Fig. 1B, C). We were able to detect TLT- 1 only in platelets, confirming that TLT-1 expression in the peripheral blood compartment in humans, as in mice, is specific for platelets.

\section{Isolation and characterization of monoclonal phage antibodies specific for TLT-I}

With the aim of defining the role of TLT- 1 in platelets and functional epitopes of this receptor, we isolated human scFvs specific for TLT-1 from a naïve library of phage-displayed scFvs. After depleting the library for clones that bound to the $\mathrm{Fc}$ fragment of $\mathrm{IgG}$, three rounds of panning against TLT-1-Fc fusion resulted in significant enrichment of phage that bound specifically to TLT-1, as assessed by ELISA, whereas no significant binding was detected with pooled phage from any of the rounds when TLT-1-Fc was replaced with the human Fc fragment alone (data not shown). A total of 180 clones were randomly picked from the phage pools from each round of selection, and 57 clones with the highest reactivity against TLT-1-Fc and no reactivity against human Fc fragment alone were selected in a phage ELISA and subjected to further characterization. These clones also showed no reactivity to bovine ubiquitin or MAR-1, a receptor with homology to TREMs and TLT-1. A representative phage ELISA for six selected clones is shown in Figure 2A. To verify that the clones bound to TLT-1 in its native conformation, phage were used in flow cytometric analysis of (HEK)293T cells transfected with TLT-1. The vast majority of phage clones $(96 \%)$ bound to TLT-1 expressing cells, as represented for clone C10 (Fig. 2B, red histogram and Fig. 2C, upper panel). No binding was detected for the naïve library (Fig. 2B, black histogram) or when clones were screened against control cells that do not express TLT-1 (Fig. 2C, bottom panel). The analysis of the deduced amino acid sequences of $\mathrm{V}_{\mathrm{H}}$ and $\mathrm{V}_{\mathrm{L}}$ revealed the presence of 24 different clones (data not shown). The complementary determining regions $(\mathrm{CDR}) 1$ and the framework regions of all clones were identical (data not shown). The CDR2 and CDR3 of $\mathrm{V}_{\mathrm{H}}$ and $\mathrm{V}_{\mathrm{L}}$ domains were the same length in all clones and showed partial sequence variability. The deduced amino acid sequences of the CDR2 and CDR3 of six selected clones are reported in Table 1. The complete DNA sequences of these scFvs are available at GenBank (accession numbers DQ375449 to DQ375454).

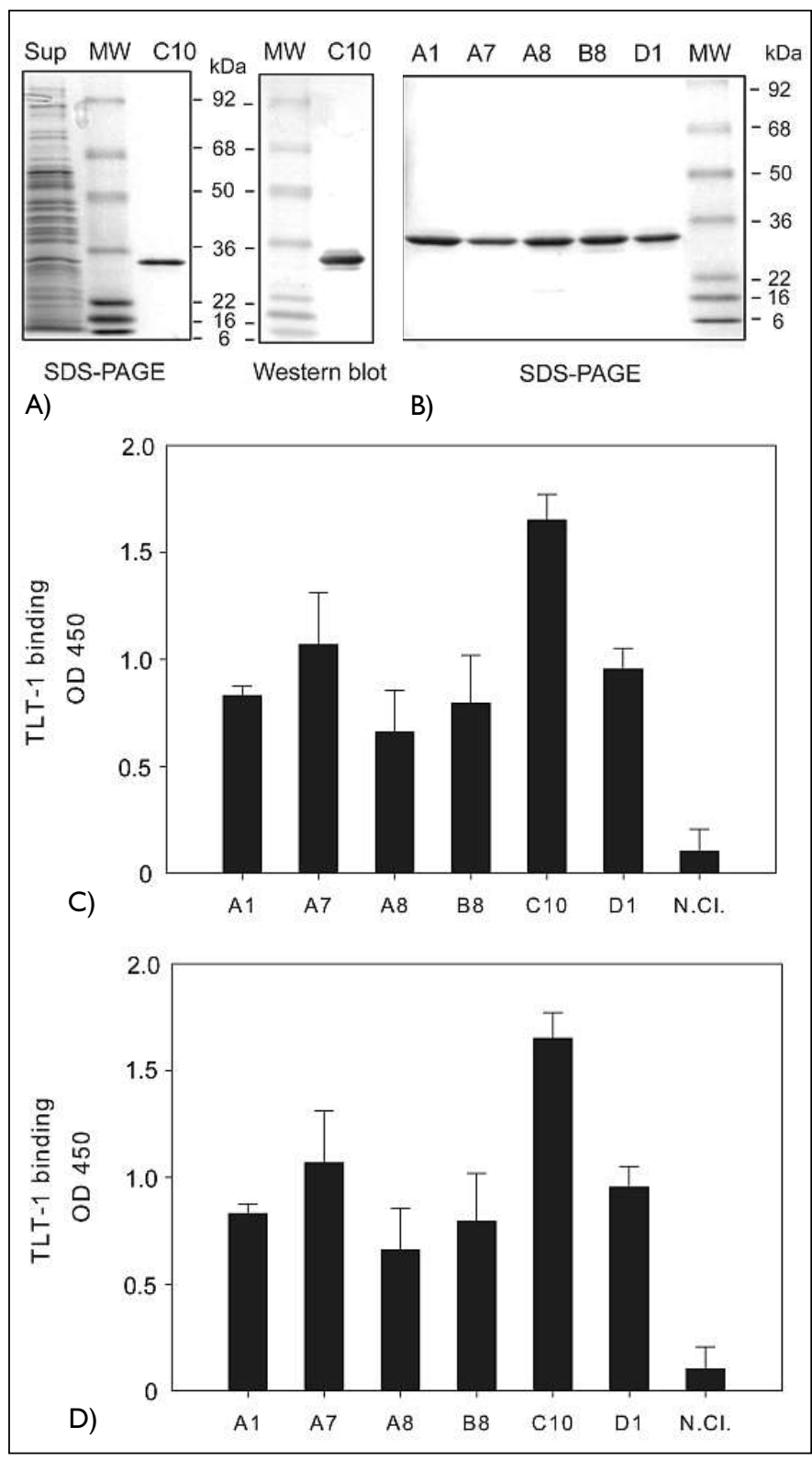

Figure 3: Purification of soluble scFvs and analysis of binding to murine TLT-I. A) Left panel: SDS-PAGE of the supernatant from CIO clone after IPTG induction (Sup, lane I, I0 $\mu$ ) and of the purified $\mathrm{Cl} 0$ $\mathrm{scFv}(\mathrm{ClO}$, lane 3, I $\mu \mathrm{g})$; right panel: Western blot of the purified scFv (CI0, lane 5) probed with an anti-His-tag antibody - HRP conjugate. Lane 2 and 4, molecular weight markers. B) SDS-PAGE of purified scFvs. C) Analysis of scFv binding to TLT-I in an ELISA assay. TLT-I-Fc was immobilized on a microtiter plate and incubated with purified anti-TLT-I scFvs or with a negative control scFv (N.Cl.) $(2 \mu \mathrm{g} /$ well). Bound scFvs were detected with Protein L-HRP conjugate. D) Analysis of TLT-I binding to scFvs in a sandwich ELISA. Purified anti-TLT-I scFvs or a negative control scFv were immobilized on a microtiter plate and incubated with TLT-I-Fc (30 ng/well). Binding of TLT-I-Fc was detected using anti-TLT-I $\mathrm{pAb}$. The data represented are the mean of three independent experiments.

\section{Production and characterization of soluble scFvs}

Selected phage clones were used to infect $E$. coli HB2151 to produce soluble scFvs. Based on the highest binding activity of the scFvs to TLT-1-Fc immobilized in an ELISA assay, six clones 
(A1, A7, A8, B8, C10, and D1) were selected for purification and further characterization (Fig. 2A). Purified scFvs showed a single band of the expected molecular weight $(28 \mathrm{kDa})$ on coomassie staining of SDS-PAGE (Fig. 3A, left panel, and Fig. 3B), which reacted on Western blot with an anti-His antibodyHRP conjugate, as shown for $\mathrm{scFv} \mathrm{C} 10$ in Figure 3A (right panel). We demonstrated binding of the purified scFvs to immobilized TLT-1-Fc in ELISA (Fig. 3C). The binding activity of scFvs was also detected by antigen-capture ELISA, immobilizing the scFvs on a plate and incubating with different amounts of TLT-1-Fc in solution (Fig. 3D). Using this method the scFv with the highest capture activity for TLT-1-Fc was $\mathrm{C} 10$ and as little as $3 \mathrm{ng} / \mathrm{well}$ of the purified protein was detectable (data not shown). The purified scFvs showed binding activity to TLT-1 transfected cells in flow cytometric analysis (data not shown).

\section{Binding of the anti-TLT-I scFvs to platelets}

To test the binding activity of the selected scFvs to TLT- 1 in the context of the platelet surface, flow cytometric analysis was performed using thrombin-activated human platelets and purified scFvs (Fig. 4A). Expression of TLT-1 on the surface of the activated platelets was detected using the anti-TLT-1 pAb (Fig. 4B, bottom panels). Resting platelets and an irrelevant $\mathrm{scFv}$, isolated from the same library, were used as negative controls. The six anti-TLT-1 scFvs reacted to a different extent with activated platelets (Fig. 4A). Strong to moderate reactivity with activated platelets was found with clones $\mathrm{C} 10, \mathrm{~A} 8, \mathrm{D} 1$ and $\mathrm{A} 1$, while scFv B8 and A7 only weakly reacted with TLT-1 on platelets. No binding of the negative control $\mathrm{scFv}$ was detected to activated platelets (Fig. 4A and B, right upper panel). All scFvs demonstrated minimal binding on resting platelets, consistent with the low level of

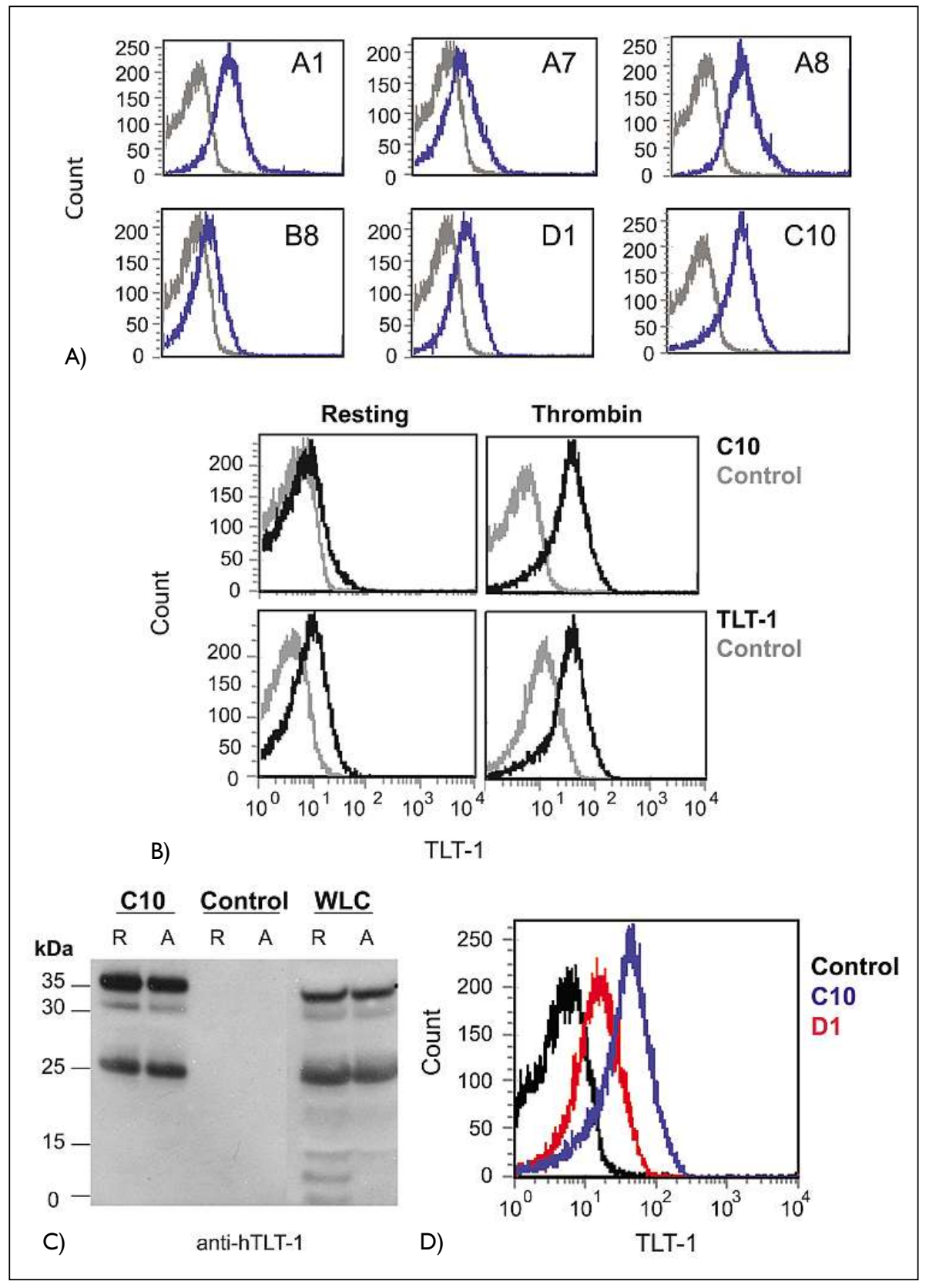

Figure 4: Binding of TLT-I specific scFvs to human platelets.

A) Purified scFvs (blue histograms) and an irrelevant scFv (grey histograms) were analyzed for their binding to thrombin-activated platelets in flow cytometry. B) Binding of scFv $\mathrm{CIO}$ (upper panels) and of anti-TLT-I pAb (lower panels) to resting and thrombin-stimulated platelets analyzed by flow cytometry. C) Western blot analysis of the immunoprecipitation of TLT-I from a human platelet lysate using scFv $\mathrm{ClO}$ and whole cell lysate $(\mathrm{WCL})$ from resting $(R)$ and thrombin-activated $(A)$ platelets. A control experiment was performed with an irrilevant scFv (Control) and in the absence of scFv (data not shown). Immunoprecipitates and platelet lysates were probed with anti-TLT-I pAb. D) Flow cytometric analysis of scFvs $\mathrm{ClO}$ and DI compared to an irrelevant scFv (control). 


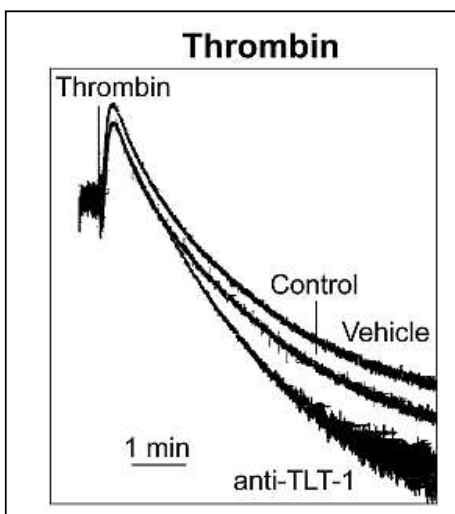

A)

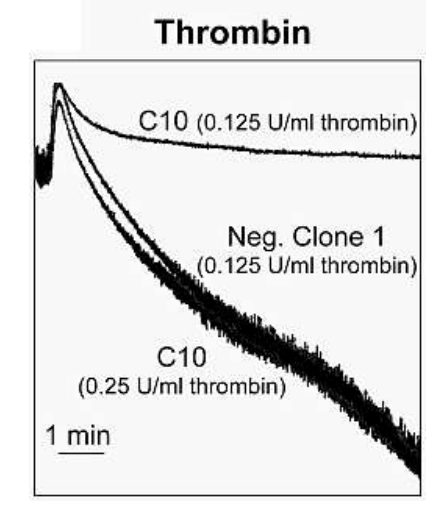

E)

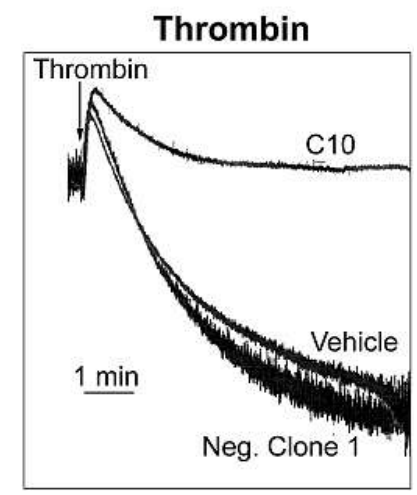

B)

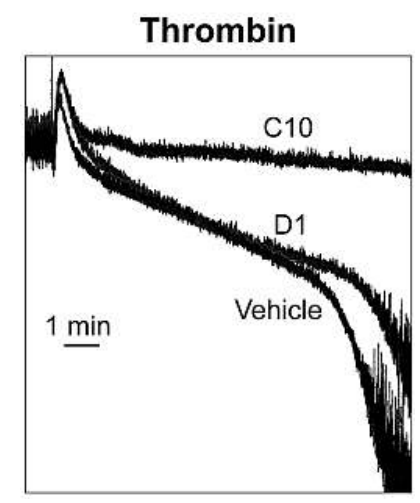

F)

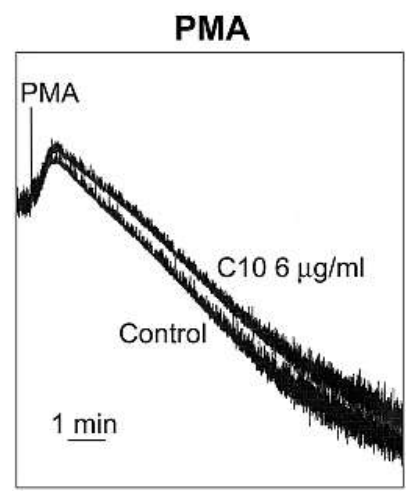

C)

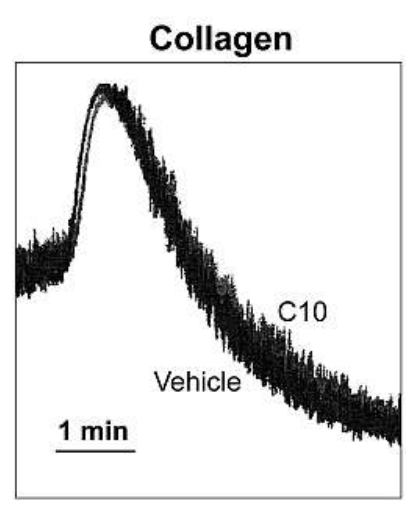

G)

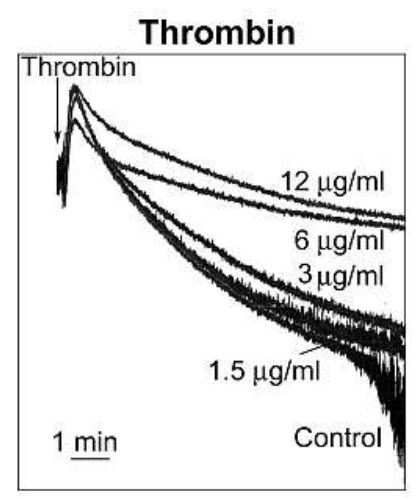

D)

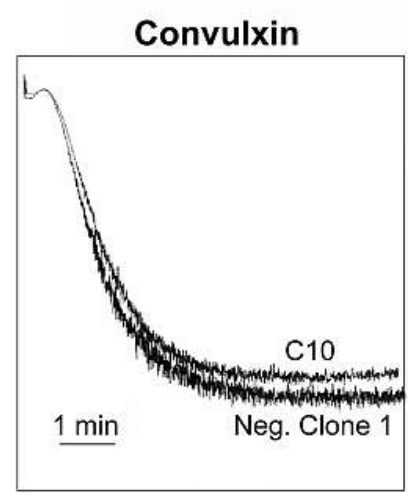

$\mathrm{H})$
Figure 5: Effect of anti-TLT-I antibodies on platelet aggregation. The effect of anti-TLT-I antibodies on platelet aggregation was analyzed in an aggregometry assay in which the inhibition of platelet aggregation is measured by the increase in absorbance. A) Effect of antiTLT-I pAb on platelet aggregation. Platelets were activated with thrombin in the presence of vehicle, anti-TLT-I pAb or control. B-H) Effect of
TLT-I specific scFvs on platelet aggregation. Washed platelets were activated with thrombin, PMA, collagen or the snake venom convulxin in the presence of vehicle, or an irrelevant scFv (Neg. clone I), or antiTLT-I scFvs ( $\mathrm{CIO}$ and $\mathrm{DI})$. Data are representative of at least three independent experiments using platelets from different donors.
TLT-1 on the surface of these cells. A representative histogram of binding to resting platelets compared to activated platelets is shown for scFv C10 in Figure 4B (upper panel).

Based on these results, the scFv that showed the highest binding activity to activated platelets compared to controls was $\mathrm{C} 10$. Moreover, the scFv C10 performed better than the commercially available pAb anti-TLT-1 (Fig. 4B). Therefore, we used scFv C10 to perform an immunoprecipitation of TLT-1 from whole cell lysates of resting or thrombin activated platelets. The scFv C10 efficiently immunoprecipitated TLT-1 from both cell lysates, as shown in the Western blot analysis of the immunoprecipitates probed with anti-hTLT-1 pAb (Fig. 4C). The two major bands of 35 and $25 \mathrm{kDa}$ detected in the Western blot analysis of platelet lysate were both present in scFv C10 immunoprecipitates, while no bands were detected in the control immunoprecipitate.

Taken together, these data confirm that we have identified scFvs highly specific for TLT- 1 and that some of these antibodies readily react with platelet TLT-1. Furthermore, this approach resulted in the isolation of scFvs with various degrees of reactivity with TLT-1 as demonstrated in Figure 4D.

\section{Inhibition of thrombin-mediated platelet aggregation by using anti-TLT-I scFvs}

Initially, we evaluated platelet aggregation using a commercially available anti-TLT- $1 \mathrm{pAb}$. We incubated platelets with antiTLT-1 pAb, anti-TREM-1 pAb as an isotype control, or vehicle alone, then added thrombin $(0.125 \mathrm{U} / \mathrm{ml})$ and measured the aggregation response. Addition of anti-TLT-1 pAb gave only a slight increase in thrombin-mediated aggregation relative to controls (Fig. 5A). This initial observation suggested that the $\mathrm{pAb}$ might not react with relevant epitopes on TLT-1. Therefore, we next tested the isolated anti-TLT-1 scFvs in the same assay. When thrombin was used as an agonist, there was a significant inhibition of aggregation by scFv C10. This inhibition was not observed using the vehicle alone or the negative control $\mathrm{scFv}$ (Fig. 5B). After thrombin stimulation, $\mathrm{scFv} \mathrm{C} 10$-treated platelets underwent initial shape change as evidenced by the immediate increase, followed by a gradual decrease in absorbance. Primary aggregation was potently suppressed in each of several donors $(\mathrm{n}=11)$. In most samples, platelets treated with $\mathrm{scFv} \mathrm{C} 10$ before the addition of thrombin finally aggregated after approximately 15-20 min (data not shown). ScFv C10 did not inhibit PMA-induced aggregation (Fig. 5C), suggesting that the TLT-1 effect can 


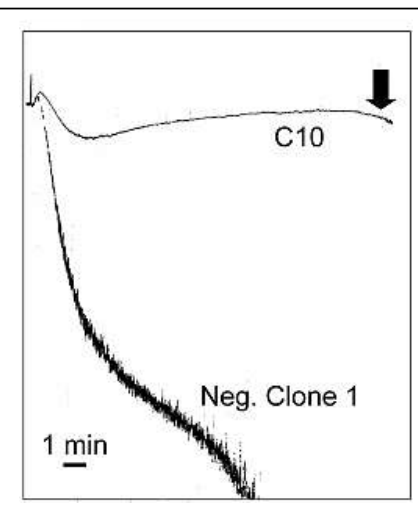

A)

Figure 6: Alpha-granule secretion in the presence of scFv CIO. A) Aggregation was initiated in the presence of $\mathrm{ClO}$, the reaction was stopped (arrow) and surface expression p-selectin was evaluated by flow cytometry. B) Histogram of p-selectin (CD62P) expression of platelets inhibited by $\mathrm{ClO}$ compared to resting and activated platelets.

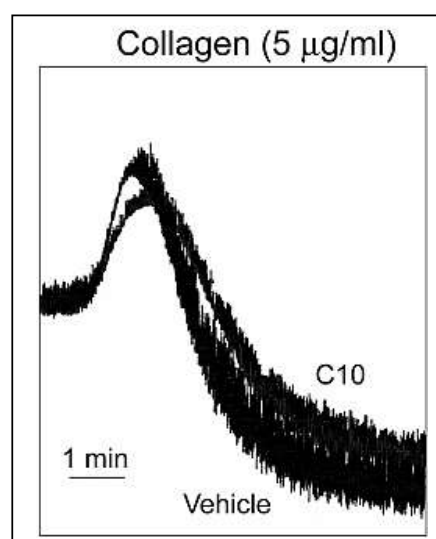

A)

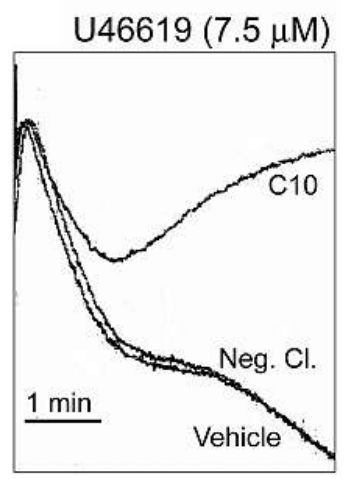

C)

D)

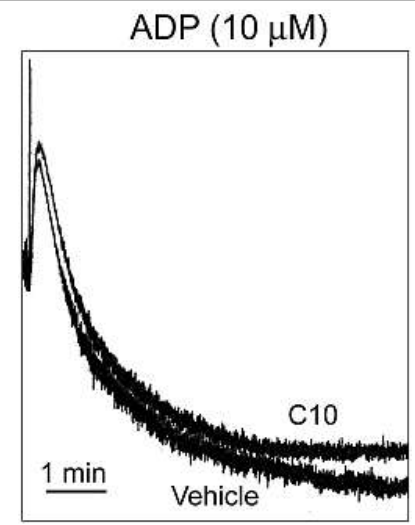

B)

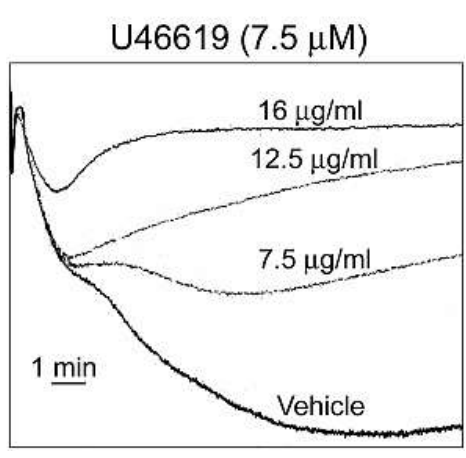

Figure 7: Effect of scFv CIO on platelet aggregation. Platelets were activated in PRP in the presence of $\mathrm{Cl} 0$ with $A$ ) collagen, $B) A D P$, $C$ and D) Thromboxane $A_{2}$ mimetic $U 466$ I 9. $D$ shows dose response using U46619.

be bypassed by direct protein kinase $\mathrm{C}$ activation, and that blockade of TLT-1 did not destroy the potential of platelets to respond. $\mathrm{ScFv}$ C10-mediated inhibition was concentration-dependent, as shown in Figure 5D. Concentrations of $1.5-3 \mu \mathrm{g} / \mathrm{ml}$ were ineffective, whereas $6 \mu \mathrm{g} / \mathrm{ml}$ gave a near maximum inhibition. Interestingly, scFv C10-mediated inhibition could be overcome by increasing concentrations of thrombin, suggesting that $\mathrm{C} 10$ affects the threshold of thrombin-mediated responsiveness (Fig. 5E). Although scFv A1 and A8 showed similar inhibitory effects as $\mathrm{scFv} \mathrm{C} 10$ (data not shown), scFv A7, B8, and D1 were ineffective even when tested at concentrations higher than required for $\mathrm{scFv}$ C10-mediated inhibition (Fig. 5F and data not shown). These results are likely due to the amino acid diversity within the CDR1 and CDR2 regions of these scFvs relative to $\mathrm{scFv} \mathrm{C} 10, \mathrm{~A} 1$ and A8 (Table 1).

Platelet aggregation can be triggered by several agonists that signal via diverse receptor systems. Therefore, we asked whether $\mathrm{scFv}$ might also be efficient at inhibiting aggregation of washed platelets induced by collagen or the GPVI-specific agonist, convulxin. We found that TLT-1 specific scFv C10 had negligible effects on aggregation induced by a range of collagen concentrations from 1-10 $\mu \mathrm{g} / \mathrm{ml}$ (Fig. 5G). Similarly, convulxin-induced aggregation was not significantly blocked by scFv $\mathrm{C} 10$ (Fig. 5H).

The majority of TLT- 1 is found within the $\alpha$-granules of resting platelets suggesting that the scFvs must act primarily after initial signaling and degranulation. Consistent with this notion, platelets stimulated with thrombin in the presence of scFvs exhibit rapid shape change consistent with primary signaling (Fig. 5B, D-F). However, we felt it important to test whether scFvs might function, in part, by blocking thrombin-induced degranulation. Therefore, we stimulated platelets in the presence of $\mathrm{scFv}$ $\mathrm{C} 10$, stopped the reaction using FACS buffer, and assessed their expression of CD62P by flow cytometry. Figure 6A shows a representative aggregation tracing denoting where cells were removed for analysis of CD62P (Fig. 6A, arrow). These experiments demonstrated that thrombin-induced CD62P expression was not decreased by $\mathrm{scFv} \mathrm{C} 10$ pretreatment even though aggregation was blocked (Fig. 6B). Because thrombin-induced CD62P is calcium-dependent, these data also demonstrate that $\mathrm{scFv}$ pretreatment does not affect proximal thrombin signaling, including calcium mobilization, and degranulation.

Because treatment with $\mathrm{scFv}$ failed to inhibit thrombin-induced shape change or degranulation, we hypothesized that the antibody might interfere with responses to secondary mediators of platelet aggregation. Therefore, we tested the effects of $\mathrm{scFv}$ pretreatment on platelet aggregation induced by ADP and thromboxane A2. In these experiments platelet aggregation was induced in PRP using collagen, ADP or the thromboxane A2 mimetic U46619 in the presence or absence of scFv C10. These experiments demonstrated that, like stimulation with collagen, ADP-induced aggregation was not affected by scFv $\mathrm{C} 10$ (Fig. 7 A, B). In contrast, aggregation induced by U46619 was efficiently blocked $\mathrm{scFv} \mathrm{C} 10$ at doses similar to those found to inhibit thrombin-induced aggregation (Fig. 7 C, D).

\section{Discussion}

Our previous work had determined that TLT-1 is an abundantly expressed, alpha-granule specific, platelet receptor in the mouse. The data we present here confirms that TLT- 1 is platelet-specific 
in humans as well. Despite the substantial levels of TLT-1 expressed in human and murine platelets and its regulation during platelet activation, until now the receptor has not been linked to any specific function. Therefore, we sought to develop a method to affect TLT-1 function in vitro with the goal of carrying any findings through to the clinic. The approach we chose was to use a phage-displayed human antibody library to derive scFvs specific for TLT-1. A repertoire of many different scFvs can be displayed on the surface of filamentous bacteriophage, allowing phage with a specific antigen-binding activity to be selected by panning on the target antigen (13). This approach has several advantages compared to the traditional hybridoma technology; i) monoclonal antibodies can be isolated faster and without the need for animal immunization (23); ii) the use of a naïve library (derived from non-immunized donors) allows the selection of antibodies against self-antigen and weakly immunogenic proteins $(17,24)$; iii) scFvs can be efficiently and economically produced in bacteria or in other expression systems $(25,26)$.

Using these monoclonal scFvs we were able to demonstrate for the first time a role for TLT-1 in platelet aggregation. Based on our aggregation traces it seems that TLT-1-mediated inhibition comes after thrombin- or U46619-induced shape change and during a period that would be described as the onset of primary aggregation. This finding is consistent with the inability of the $\mathrm{scFv}$ to activate platelet aggregation (data not shown). AntiTLT- $1 \mathrm{scFv}$ inhibition would, therefore, not be expected to take effect until after initial activation had released TLT-1 from the $\alpha$-granules. Interestingly, two of the identified clones (C10 and D1) showed fairly strong recognition of TLT-1 by flow cytometric analysis (Fig. 4D) but variable levels of inhibition on platelet aggregation (Fig. 5F). This variance may reflect the differences in binding characteristics exemplified by the flow cytometry data. An alternative possibility is that these scFvs bind different epitopes with the region bound by $\mathrm{C} 10$ being more important for activation. To understand these distinctions scFv epitope mapping and/or direct measurement of their affinity for TLT-1 would be required.

TLT-1 is an ITIM containing receptor demonstrated to bind SHP-1 and/or SHP-2 (12). Classical models of ITIM-mediated inhibition would suggest that TLT-1 might inhibit an ITAM containing, phosphotyrosine-based receptor system such as the collagen receptor GPVI, which signals via FcR $\gamma$. In addition, most models of inhibitory signaling require co-clustering of the inhibitory and target receptors to promote inhibitory signaling. ScFvs are small, monovalent molecules, making co-clustering unlikely. We cannot rule out, however, that at high enough concentrations, the $\mathrm{scFv}$ engages enough TLT-1 molecules to send an inhibition signal. A more likely interpretation of our data is the scFv blocking TLT-1-ligand interactions during aggregation. Our use of washed platelets here would imply that the TLT-1 ligand is either on platelets or at least stored in platelets at sufficient concentrations to become biologically active upon platelet degranulation. This type of co-activator model for TLT-1 function would be consistent with data showing that co-engagement of TLT-1 with the Fc receptor of RBL cells enhances calcium mobilization in an ITIM-dependent fashion (12). These authors sug- gest this co-activator activity was due to ITIM-mediated recruitment of SHP-2 to the receptor complex.

Where then might TLT-1 signals intersect with those of thrombin or $\mathrm{TxA}_{2}$ The specific anti-TLT-1 scFv inhibition of $\mathrm{TxA}_{2}-$ and thrombin-mediated aggregation, relative to that induced by ADP, suggests a potential integration point. Although ADP, TxA $\mathrm{A}_{2}$ and thrombin are all coupled to G-protein signaling pathways, ADP utilizes the $G_{\alpha \mathrm{i}}$ or $\mathrm{G}_{\alpha \mathrm{q}}$ pathways whereas both thrombin and $T x A_{2}$ couple to $G_{\alpha 12 / 13}$ (27). This suggests that TLT-1 signaling during aggregation may specifically synergize with $\mathrm{G}_{\alpha 12 / 13}$ driven pathways such as the activation of the small GTPase RhoA. Interestingly, $\mathrm{G}_{\mathrm{a} 12 / 13}$ signaling is known to involve tyrosine phosphorylation, and Rho activation can be enhanced by phosphatases such as SHP-2 (27-30). It is therefore tempting to speculate that TLT-1 may in someway regulate the activation of RhoA pathways during platelet aggregation. Direct investigation of these specific signaling pathways will require substantial additional studies well outside of the scope of the current study.

Regardless of their biochemical mechanism of action, antiTLT-1 scFv, like the anti-integrin $\alpha_{\text {IIb }} \beta_{3}$ scFvs described by Schwartz et al. (31), have several features that make them promising candidates for the development of anti-thrombotic drugs. scFvs are of complete human origin avoiding major immunological imcompatabilities. In addition, their small size should result in favorable pharmacological properties such as faster elimination with consequent reductions of bleeding complications and better penetration of thrombi. Lastly, should it be required, $\mathrm{scFv}$ are easy to engineer to increase affinity or construct derivatives while retaining antigen specificity.

In summary, we demonstrate the isolation, characterization, and utility of scFv monoclonal antibodies that recognize TLT-1. Our application of these antibodies in in vitro assays of human platelet function has, for the first time, defined a platelet-specific function for TLT-1 in the regulation of aggregation. Regardless of the mechanisms that responsible for inhibition, these findings open the possibility for the use of these and/or related TLT-1 specific agents for therapeutic intervention in a variety of human disorders associated with thrombosis and coagulation.

\section{Acknowledgments}

We thank MRC Geneservice for its kindly supply and permission to use the Human Single Fold scFv libraries I + J (Tomlinson I + J). We thank Pat M. Martin (SAIC-Frederick, Laboratory of Genomic Diversity) for sequencing of the clones, and Maura Calvani and Giovanni Melillo (Developmental Therapeutics Program, NCI-Frederick) for providing the human vascular endothelial cells. We also thank Lymar Morrell, Rosa Cantras, UCC staff, Jose Rodriguez, Eddie Rios, and Grissel Font for providing the tools to complete these experiments at UCC. This research was supported in part by the Intramural Research Program of the NIH, National Cancer Institute, Center for Cancer Research, and by Grant Number 2G12RR3035 from the National Center for Research Resources (NCRR), a Component of the NIH. By acceptance of this article, the publisher or recipient acknowledges the right of the U.S. Government to retain a nonexclusive, royalty-free license in and to any copyright of the article. The content of this publication does not necessarily reflect the views or policies of the department of Health and Human Services, nor does mention of trade names, commercial products, or organizations imply endorsement by the U.S. government. 


\section{References}

1. Ruggeri ZM. Platelets in atherothrombosis. Nat Med 2002; 8: 1227-1234.

2. Burke AP, Farb A, Malcom GT, et al. Coronary risk factors and plaque morphology in men with coronary disease who died suddenly. N Engl J Med 1997; 336: 1276-1282.

3. Virmani R, Kolodgie FD, Burke AP, et al. Lessons from sudden coronary death: a comprehensive morphological classification scheme for atherosclerotic lesions. Arterioscler Thromb Vasc Biol 2000; 20: $1262-1275$.

4. Phillips DR, Conley PB, Sinha U, et al. Therapeutic approaches in arterial thrombosis. J Thromb Haemost 2005; 3: 1577-1589.

5. Nieswandt B, Aktas B, Moers A, et al. Platelets in atherothrombosis: lessons from mouse models. J Thromb Haemost 2005; 3: 1725-1736.

6. A randomised, blinded, trial of clopidogrel versus aspirin in patients at risk of ischaemic events (CAPRIE). CAPRIE Steering Committee. Lancet 1996; 348: 1329-1339.

7. Popma JJ, Satler LF. Early and late clinical outcome following coronary angioplasty performed with platelet glycoprotein IIb/IIIa receptor inhibition: the EPIC Trial results. J Invasive Cardiol 1994; 6 (Suppl A): 19A-28A.

8. Genetta TB, Mauro VF. ABCIXIMAB: a new antiaggregant used in angioplasty. Ann Pharmacother 1996; 30: 251-257.

9. Washington AV, Quigley L, McVicar DW. Initial characterization of TREM-like transcript (TLT)-1: a putative inhibitory receptor within the TREM cluster. Blood 2002; 100: 3822-3824.

10. Washington AV, Schubert RL, Quigley L, et al. A TREM family member, TLT-1, is found exclusively in the alpha-granules of megakaryocytes and platelets. Blood 2004; 104: 1042-1047.

11. Radaev S, Kattah M, Rostro B, et al. Crystal structure of the human myeloid cell activating receptor TREM-1. Structure 2003; 11: 1527-1535.
12. Barrow $\mathrm{AD}$, Astoul $\mathrm{E}$, Floto $\mathrm{A}$, et al. Cutting edge: TREM-like transcript-1, a platelet immunoreceptor tyrosine-based inhibition motif encoding costimulatory immunoreceptor that enhances, rather than inhibits, calcium signaling via SHP-2. J Immunol 2004; 172: 5838-5842.

13. Winter G, Griffiths AD, Hawkins RE, et al. Making antibodies by phage display technology. Annu Rev Immunol 1994; 12: 433-455.

14. McCafferty J, Griffiths AD, Winter G, et al. Phage antibodies: filamentous phage displaying antibody variable domains. Nature 1990; 348: 552-554.

15. Bird RE, Hardman KD, Jacobson JW, et al. Singlechain antigen-binding proteins. Science 1988; 242: 423-6.

16. 16. Laffly E, Sodoyer R. Monoclonal and recombinant antibodies, 30 years after ... Hum Antibodies 2005; 14 : 33-55

17. Griffiths AD, Malmqvist M, Marks JD, et al. Human anti-self antibodies with high specificity from phage display libraries. EMBO J 1993; 12: 725-734

18. Goletz S, Christensen PA, Kristensen P, et al. Selection of large diversities of antiidiotypic antibody fragments by phage display. J Mol Biol 2002; 315: 1087-1097.

19. Yan JP, Ko JH, Qi YP. Generation and characterization of a novel single-chain antibody fragment specific against human fibrin clots from phage display antibody library. Thromb Res 2004; 114: 205-211.

20. Liu ZX, Yi GH, Qi YP, et al. Identification of singlechain antibody fragments specific against SARS-associated coronavirus from phage-displayed antibody library. Biochem Biophys Res Commun 2005; 329: 437-444.

21. Paul SP, Taylor LS, Stansbury EK, et al. Myeloid specific human $\mathrm{CD} 33$ is an inhibitory receptor with differential ITIM function in recruiting the phosphatases SHP-1 and SHP-2. Blood 2000; 96: 483-490.

22. Gattis JL, Washington AV, Chisholm MM, et al. The structure of the extracellular domain of triggering re- ceptor expressed on myeloid cells like transcript - 1 , and evidence for a naturally occuring soluble fragment. J Biol Chem 2006; 281: 13396-13403.

23. Hoogenboom HR, Winter G. By-passing immunisation. Human antibodies from synthetic repertoires of germline $\mathrm{VH}$ gene segments rearranged in vitro. $\mathrm{J} \mathrm{Mol}$ Biol 1992; 227: 381-388.

24. Vaughan TJ, Williams AJ, Pritchard K, et al. Human antibodies with sub-nanomolar affinities isolated from a large non-immunized phage display library. Nat Biotechnol 1996; 14: 309-314.

25. Miller KD, Weaver-Feldhaus J, Gray SA, et al. Production, purification, and characterization of human $\mathrm{scFv}$ antibodies expressed in Saccharomyces cerevisiae, Pichia pastoris, and Escherichia coli. Protein Expr Purif 2005; 42: 255-267.

26. Feldhaus MJ, Siegel RW. Yeast display of antibody fragments: a discovery and characterization platform. J Immunol Methods 2004; 290: 69-80.

27. Offermanns S. Activation of platelet function through $\mathrm{G}$ protein-coupled receptors. Circ Res 2006; 99: 1293-1304.

28. Klages B, Brandt U, Simon MI, et al. Activation of G12/G13 results in shape change and Rho/Rho-kinasemediated myosin light chain phosphorylation in mouse platelets. J Cell Biol 1999; 144: 745-754.

29. Moon SY, Zheng Y. Rho GTPase-activating proteins in cell regulation. Trends Cell Biol 2003; 13 . $13-22$.

30. Kontaridis MI, Eminaga S, Fornaro M, et al. SHP-2 positively regulates myogenesis by coupling to the Rho GTPase signaling pathway. Mol Cell Biol 2004; 24 5340-5352.

31. Schwarz M, Rottgen P, Takada Y, et al. Single-chain antibodies for the conformation-specific blockade of activated platelet integrin alphaIlbbeta3 designed by subtractive selection from naive human phage libraries. FASEB J 2004; 18: 1704-1706. 